\title{
Edge Reduction for EVMDDs to Speed Up Analysis of Multi-State Systems
}

\author{
Shinobu Nagayama* $\quad$ Tsutomu Sasao ${ }^{\dagger} \quad$ Jon T. Butler ${ }^{\ddagger} \quad$ Mitchell A. Thornton ${ }^{\S} \quad$ Theodore W. Manikas ${ }^{\S}$ \\ *Dept. of Computer and Network Eng., Hiroshima City University, Hiroshima, JAPAN \\ ${ }^{\dagger}$ Dept. of Computer Science, Meiji University, Kawasaki, JAPAN \\ †Dept. of Electr. and Comp. Eng., Naval Postgraduate School, Monterey, CA USA

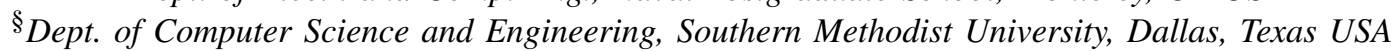

\begin{abstract}
This paper proposes a new reduction rule for edge-valued multi-valued decision diagrams (EVMDDs), which improves the speed of analysis of multi-state systems (MSSs). Existing reduction rules for decision diagrams remove redundant nodes, while the proposed rule removes redundant edges in EVMDDs. Since the time to do an analysis in an MSS depends on the number of edges in the EVMDD, the proposed rule is faster especially when used with edge minimization algorithms based on variable grouping. Experimental results show that the proposed rule reduces the number of edges by up to $30 \%$, and this results in an analysis time that is reduced by up to $30 \%$.
\end{abstract}

Keywords-Reduction rules for decision diagrams; EVMDDs; edge reduction of EVMDDs; multi-state systems; system analysis based on decision diagrams.

\section{INTRODUCTION}

A multi-state system (MSS) [8], [9] is a model in which performance, reliability, safety, efficiency, power consumption, etc. are represented by states. It is widely used to model various fault tolerant systems including computer server systems, telecommunication systems, water, gas, electrical power distribution systems, flight control systems, and nuclear power plant monitoring systems [3]-[5], [16], [19], [21], [23], [24]. To design dependable fault tolerant systems, intensive analysis of MSSs in terms of various assessment measures, such as reliability and availability is needed [16]. An approach to solving this complex problem is to compute the probability of each state of an MSS [21], [23]. This is because many measures can be easily computed from the state probabilities [1], [8], [9]. However, assessing the state probabilities is time-consuming.

For fast assessment of the state probabilities, many methods based on binary decision diagrams (BDDs) [2]-[4], [6], [23] and multi-valued decision diagrams (MDDs) [10], [13], [20]-[22] have been proposed, and they have attracted much attention in recent years. Especially, MDD based methods hold promise, as [17] showed that an MDD based method is more efficient than a BDD based one.

Most of these methods assume only stationary probability distributions for analysis, and thus, they cannot accommodate with probability distributions that depend on time. This is because only steady-state analysis of systems is important in many practical systems (especially in systems working for a long time). However, in safety-critical systems such as flight control systems and nuclear power plant monitoring systems, not only steady-state analysis but also transient analysis should be done.

Although methods based on the Markov model [5], [8], [9], [19] can deal with probability distributions that depend on time, they are impractical for a large MSS. This is because their time complexity is $O\left(m^{3 n}\right)$, where $m$ is the number of states, and $n$ is the number of components in an MDD [3], [4]. For faster transient analysis of large MSSs, an MDD based method has been proposed [18]. This method can make a transient analysis in the same way as a steadystate analysis by assigning dynamic probability distributions to edges in an MDD. However, even this method is slow, since for a transient analysis, a system has to be analyzed many times while advancing time by a small step. Thus, a faster analysis method is desired.

In this paper, we propose a new reduction rule for edgevalued MDDs (EVMDDs) [12], [13] to speed up the analysis of MSSs. The existing reduction rules of decision diagrams are intended to reduce redundant nodes, but the proposed rule is intended to reduce redundant edges in EVMDDs. Since analysis time of MSSs depends on the number of edges in an EVMDD, the proposed edge reduction rule can also reduce analysis time. The proposed technique can be applied to a wide range of analyses including steady-state analysis, transient analysis, and analysis of systems having dependent components [14]. This is because the proposed technique is a fundamental one.

This paper is organized as follows: Section II defines MSSs, EVMDDs, and variable grouping. Section III introduces the analysis method of MSSs using EVMDDs, and in Section IV, we propose an edge reduction of EVMDDs, and an analysis method using EVMDDs with reduced edges. Experimental results are shown in Section V.

\section{PRELIMinaries}

This section defines MSSs, structure functions, EVMDDs to represent structure functions, and variable grouping.

\section{A. Multi-State Systems and Structure Functions}

Definition 1: A multi-state system (MSS) is a model of a system that represents, as states, a capability, such as 


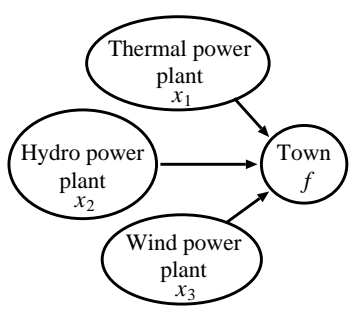

(a) MSS.

\begin{tabular}{|ccc|c|}
\hline$x_{1}$ & $x_{2}$ & $x_{3}$ & $f$ \\
\hline 0 & 0 & 0 & 0 \\
0 & 0 & 1 & 1 \\
0 & 0 & 2 & 1 \\
0 & 1 & 0 & 1 \\
0 & 1 & 1 & 2 \\
& $\vdots$ & & $\vdots$ \\
2 & 2 & 2 & 5 \\
\hline
\end{tabular}

(b) Structure function.
Figure 1. MSS for an electrical power distribution system and its structure function [15].

performance, capacity, or reliability. There are usually more than two states, and so a multiple-valued analysis is required. When components in a system are modeled as well, it is called a MSS with multi-state components. In this paper, it is simply called an MSS.

Definition 2: A state of an MSS depends only on states of components in the system. A system with $n$ components can be considered as a multi-valued function $f\left(x_{1}, x_{2}, \ldots, x_{n}\right)$ $: R_{1} \times R_{2} \times \ldots \times R_{n} \rightarrow M$, where each $x_{i}$ represents a component with $r_{i}$ states, $R_{i}=\left\{0,1, \ldots, r_{i}-1\right\}$ is a set of the states, and $M=\{0,1, \ldots, m-1\}$ is a set of the $m$ system states. This multi-valued function is called a structure function of the MSS.

Example 1: Fig. 1(a) shows an MSS for an electrical power distribution system. In this figure, the thermal power plant $x_{1}$, the hydro power plant $x_{2}$, and the wind power plant $x_{3}$ have three states which correspond to supply levels: 0 (breakdown), 1 (partially supply), and 2 (full supply). And, the system has six states which correspond to the percentage of area of a town that is blacked out: 0 (complete blackout), 1 (90\% blackout), 2 (60\% blackout), 3 (30\% blackout), 4 (10\% blackout), and 5 ( $0 \%$ blackout).

In this way, by assigning a value to each state, we obtain the 6-valued structure function $f$ shown in Fig. 1(b). Note that Fig. 1(b) shows a part of the $3^{3}=27$ entry table since it is too large to be included in its entirety. (End of Example)

\section{B. Edge-Valued Multi-Valued Decision Diagrams}

Definition 3: An edge-valued multi-valued decision diagram (EVMDD) [12] is an extension of the MDD [7], [11], and represents a multi-valued function. It consists of one terminal node representing 0 and non-terminal nodes with edges having integer weights; 0-edges always have zero weights. In the EVMDD, the following two reduction rules are applied:

1) Share equivalent sub-graphs.

2) Delete a non-terminal node satisfying the two conditions: 1) its outgoing edges all point to the same node $v$, and 2) all the edges have 0 weights. And, redirect edges, which point to the deleted node, to $v$

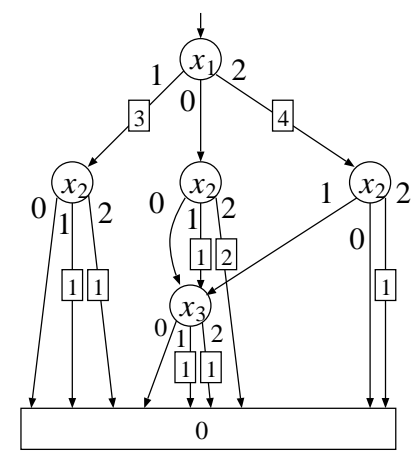

Figure 2. EVMDD for the structure function [15].

In the EVMDD, the function value is represented as a sum of weights of edges traversed from the root node to the terminal node.

Example 2: Fig. 2 shows an EVMDD for the structure function of Example 1.

(End of Example)

\section{Variable Grouping}

Definition 4: Let $X=\left(x_{1}, x_{2}, \ldots, x_{n}\right)$ be an ordered set of $n$ multi-valued variables. Partition $X$ into $u$ partially ordered subsets as follows:

$$
\begin{aligned}
X_{1}= & \left(x_{1}, x_{2}, \ldots, x_{k_{1}}\right) \\
X_{2}= & \left(x_{k_{1}+1}, x_{k_{1}+2}, \ldots, x_{k_{1}+k_{2}}\right) \\
& \vdots \\
X_{u}= & \left(x_{k_{1}+k_{2}+\ldots+1}, x_{k_{1}+k_{2}+\ldots+2}, \ldots, x_{n}\right) .
\end{aligned}
$$

Then, $\left(X_{1}, X_{2}, \ldots, X_{u}\right)$ is a grouping of $X$. Each ordered set $X_{i}=\left(x_{j+1}, x_{j+2}, \ldots, x_{j+k_{i}}\right)$ forms a composite variable whose domain is $\left\{0,1, \ldots, r_{j+1} \times r_{j+2} \times \ldots \times r_{j+k_{i}}-1\right\}$, where $\left|X_{i}\right|=k_{i}$ and $k_{1}+k_{2}+\ldots+k_{u}=n$. Note that the order of the original multi-valued variables is preserved in a grouping.

By considering each composite variable $X_{i}$ as a larger-valued variable, the original multi-valued function $f\left(x_{1}, x_{2}, \ldots, x_{n}\right): R_{1} \times R_{2} \times \ldots \times R_{n} \rightarrow M$ can be converted into another multi-valued input function $g\left(X_{1}, X_{2}, \ldots, X_{u}\right)$ : $P_{1} \times P_{2} \times \ldots \times P_{u} \rightarrow M$, where $P_{i}=\left\{0,1, \ldots, r_{j+1} \times r_{j+2} \times\right.$ $\left.\ldots \times r_{j+k_{i}}-1\right\}$.

In this paper, for convenience, an EVMDD representing the function $g$ obtained by grouping variables is called a GEVMDD.

Example 3: When the multi-valued variables $x_{1}, x_{2}, x_{3}$ in Example 1 are grouped into two composite variables, we have

$$
X_{1}=\left(x_{1}, x_{2}\right) \text { and } X_{2}=\left(x_{3}\right)
$$

Note that since $x_{1}$ and $x_{2}$ are 3 -valued variables, the composite variable $X_{1}$ consisting of $x_{1}$ and $x_{2}$ is a 9-valued variable. 


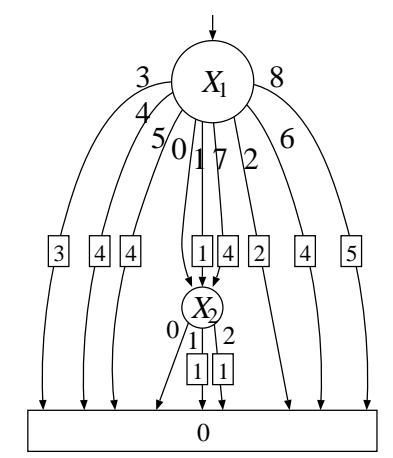

Figure 3. GEVMDD for the function $g\left(X_{1}, X_{2}\right)$ [15].

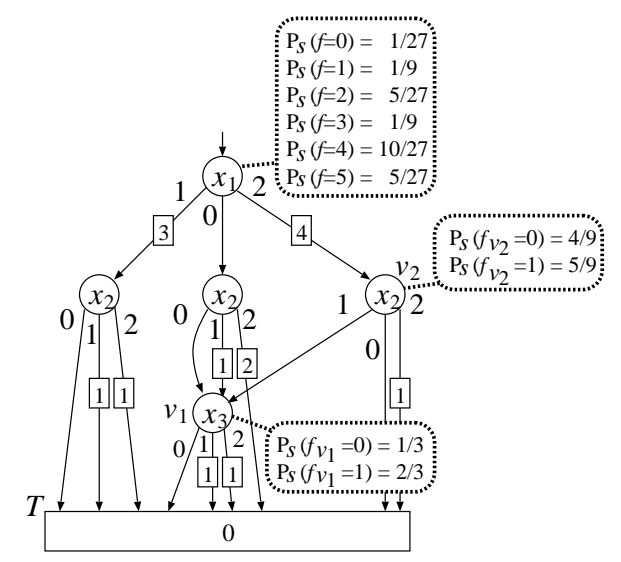

Figure 4. Analysis of the MSS using EVMDD [15].

The GEVMDD representing the obtained function $g\left(X_{1}, X_{2}\right)$ is shown in Fig. 3.

(End of Example)

\section{AnAlysis Method Using EVMDDs}

\section{A. Steady-State Analysis of Multi-State Systems}

Definition 5: The probability that a structure function $f$ has the value $s$ is denoted by $P_{s}(f=s)$, where $s \in$ $\{0,1, \ldots, m-1\}$. The probability that a component $x_{i}$ has the value $c$ is denoted by $P_{c}\left(x_{i}=c\right)$, where $c \in\left\{0,1, \ldots, r_{i}-1\right\}$.

An analysis of MSSs solves the following:

Problem 1: Given a structure function $f$ of an MSS and the probability of each state of each component $P_{c}\left(x_{i}=c\right)$, compute the probability of each state of the MSS $P_{s}(f=$ $s)$. For simplicity, we assume that the probabilities of all component states are independent of each other.

To solve this problem efficiently, a method using EVMDDs has been proposed [13]. The method represents given structure functions using EVMDDs, and computes probabilities for a structure function by merging probabilities for sub-functions represented by nodes in a bottom-up manner.
Example 4: Let us compute the probability of each state of the MSS using the EVMDD in Fig. 4. In this example, we assume that all states of each component occur with the same probability, $1 / 3$.

First, we have $P_{S}\left(f_{T}=0\right)=1$ at the terminal node $T$. Then, we compute probabilities for a sub-function $f_{v_{1}}$ at node $v_{1}$. Since this node has two edges pointing to $T$ whose values are 1 , and the two edges represent $f_{v_{1}}=1$, we have

$$
\begin{aligned}
P_{s}\left(f_{T}=0\right) \times P_{c}\left(x_{3}=1\right)= & 1 / 3, \\
P_{s}\left(f_{T}=0\right) \times P_{c}\left(x_{3}=2\right)= & 1 / 3, \text { and thus, } \\
P_{S}\left(f_{v_{1}}=1\right)= & P_{S}\left(f_{T}=0\right) \times P_{c}\left(x_{3}=1\right) \\
& +P_{s}\left(f_{T}=0\right) \times P_{c}\left(x_{3}=2\right) \\
= & 2 / 3 .
\end{aligned}
$$

Thus, $P_{s}\left(f_{v_{1}}=0\right)=1 / 3$ and $P_{s}\left(f_{v_{1}}=1\right)=2 / 3$ for $v_{1}$. At $v_{2}$, the probabilities at the terminal node and $v_{1}$ are multiplied by $1 / 3$, and they are merged. Thus, $P_{s}\left(f_{v_{2}}=0\right)=4 / 9$ and $P_{S}\left(f_{v_{2}}=1\right)=5 / 9$. Similarly, by performing the same computation at each node in a bottom-up manner, we have the following at the root node: $P_{s}(f=0)=1 / 27, P_{s}(f=1)=$ $1 / 9, P_{s}(f=2)=5 / 27, P_{s}(f=3)=1 / 9, P_{s}(f=4)=10 / 27$, and $P_{s}(f=5)=5 / 27$.

(End of Example)

\section{B. Transient Analysis of Multi-State Systems}

In steady-state analysis, it is assumed that the probabilities of component states $P_{c}\left(x_{i}=c\right)$ are constants, as shown in the previous subsection, since those probabilities converge to constant values after enough time goes by, as will be shown later. On the other hand, transient analysis assumes dynamic probability distributions depending on time, such as Weibull distributions that are widely used for modeling reliability of components considering their aging degradation. That is, in transient analysis, probabilities of component states $P_{c}\left(x_{i}=\right.$ c) are given as functions of time.

However, by assigning function values at a certain time to edges, we can apply the same analysis method to transient analysis as well. And, by analyzing a system many times while advancing time in a small step, we can perform transient analysis of an MSS [18].

Example 5: Make the following three assumptions: 1) the thermal power plant $x_{1}$ in Fig. 1 consists of two identical power generators that work independently each other; 2) the probability that a power generator fails is given as a cumulative distribution function (CDF) of a Weibull distribution:

$$
1-e^{-(t / 5)^{2}}
$$

where $t$ is time; and, 3) $60 \%$ of failures occurring at a power generator are repairable.

Then, the probabilities whether a power generator (PG) works $(\mathrm{PG}=1)$ or not $(\mathrm{PG}=0)$ can be computed using a decision diagram in Fig. 5(a). In this figure, the variable $w$ represents whether the power generator works $(w=1)$ or fails $(w=0)$, and $r$ represents whether a failure is repairable 


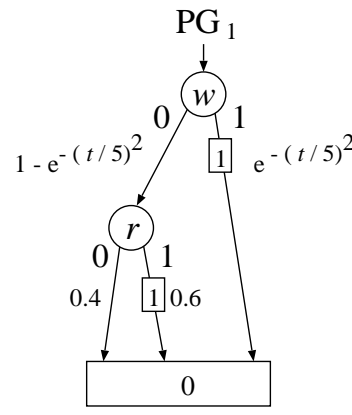

(a) Power generator.

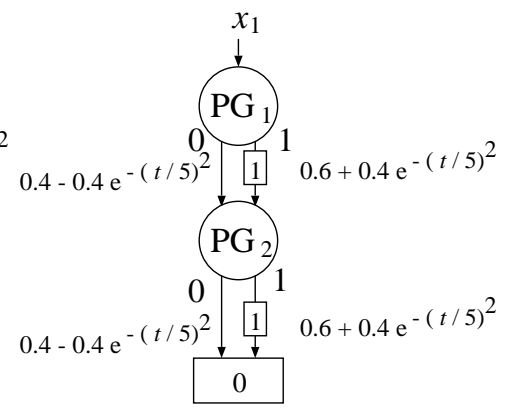

(b) Thermal power plant.
Figure 5. EVMDDs for a power generator and the thermal power plant.

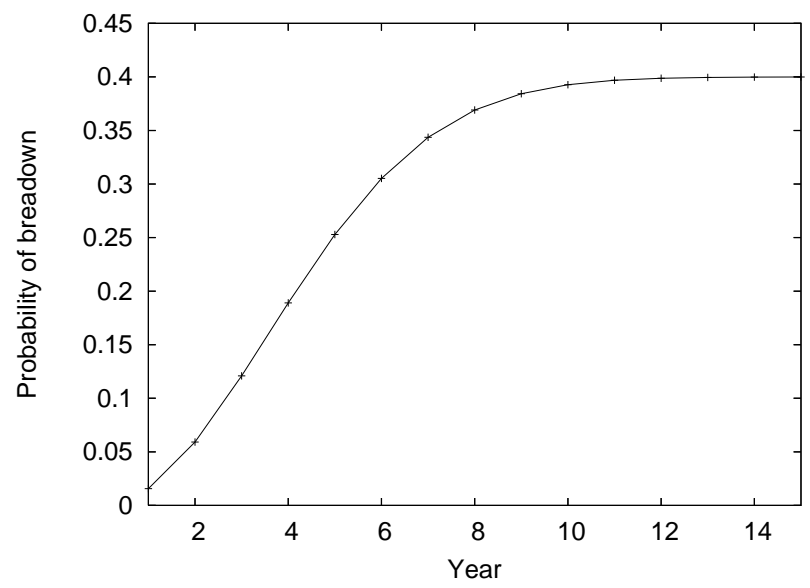

Figure 6. Result of transient analysis of a power generator.

$(r=1)$ or not $(r=0)$. Similarly, the probability of various states of the thermal power plant $x_{1}$ can be computed by using a decision diagram in Fig. 5(b) and analysis results of power generators $\mathrm{PG}_{1}$ and $\mathrm{PG}_{2}$. In this way, by assigning function values at a certain time to edges, and by analyzing system components hierarchically while advancing time by a small step, we can perform transient analysis of the whole power distribution system in Fig. 1.

Figs. 6 and 7 show results of transient analysis of a power generator and the thermal power plant $x_{1}$, respectively. From these figure, we can see that probabilities converge to constant values after enough time goes by. In steady-state analysis, these converged values are used. (End of Example)

Since for a transient analysis, a system has to be analyzed many times while advancing time by a small step, a faster analysis method is desired.

\section{EdGe Reduction of EVMDDs}

Since the computation time of EVMDD based analysis method depends on the number of edges in an EVMDD, minimization of the number of edges by variable grouping

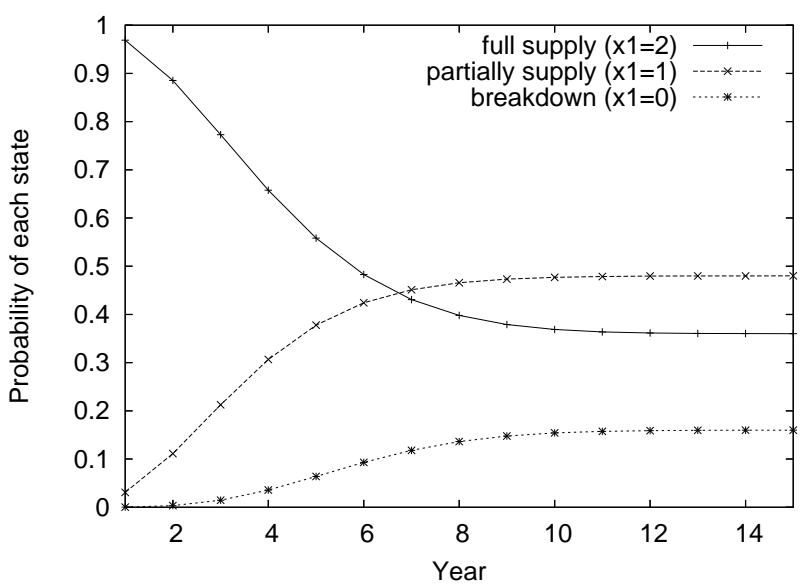

Figure 7. Result of transient analysis of the thermal power plant $x_{1}$.

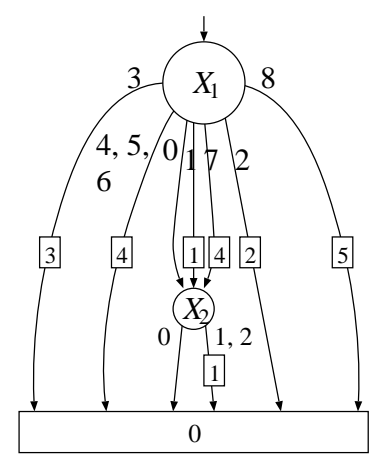

Figure 8. Reduced GEVMDD for the function $g\left(X_{1}, X_{2}\right)$.

is helpful to reduce analysis time [15]. However, variable grouping tends to increase the number of redundant edges that point to the same node and have the same edge value, as shown in Fig. 3. The GEVMDD has redundant edges for $X_{1}=4,5,6$ and $X_{2}=1,2$. We make the analysis faster by reducing these redundant edges.

To reduce such redundant edges, we propose the following reduction rule, and add it to the reduction rules of EVMDDs.

- Merge equivalent multiple edges, which leave from the same node, point to the same node, and have the same edge values, into a single edge, and share the edge among corresponding values of an input variable.

In this paper, for convenience, an EVMDD and a GEVMDD whose edges are reduced are called an REVMDD and an RGEVMDD, respectively.

Example 6: Fig. 8 shows the reduced GEVMDD (RGEVMDD) of the GEVMDD in Fig. 3. This RGEVMDD has 9 edges while the original GEVMDD has 12 edges.

(End of Example)

To analyze an MSS using an RGEVMDD, we begin by 


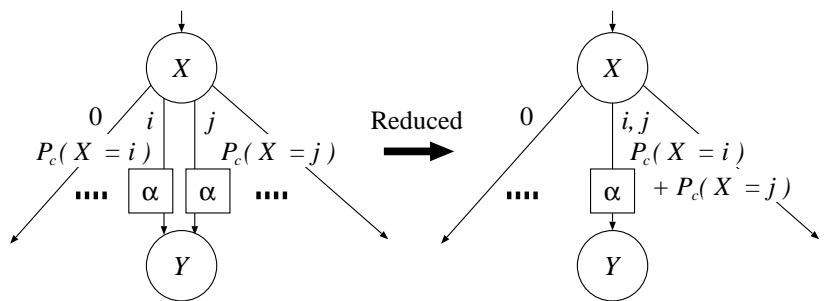

Figure 9. Probability of shared edge.

assigning probabilities of component states to edges. Shared edges are assigned sums of probabilities corresponding to merged edges, as shown in Fig. 9. Then, the analysis method shown in Section III is applied to the RGEVMDD.

Since edge reduction is a fundamental technique, it can be used along with the hybrid analysis method [14] that is used for analysis of systems having dependent components.

\section{EXPERIMENTAL RESULTS}

To show the efficiency of the proposed edge reduction technique, we used the same analysis algorithms and the same structure functions as [13], [14]. The methods are implemented on our private EVMDD package, and run on the following computer environment: CPU: Intel Core 2 Quad Q6600 2.4GHz, memory: 4GB, OS: CentOS 5.7, and C-compiler: gcc -O3 (version 4.1.2). Table I shows the number of edges in various EVMDDs for randomly generated $m$-state systems with $n 3$-state components. In this table, the columns "Grouping" and "Reduction" show computation times of the edge minimization algorithm [15] and edge reduction presented in Section IV, respectively.

From this table, we can see that even ordinary EVMDDs have redundant edges, but GEVMDDs have more redundant edges. Thus, edge reduction works more effectively when it is used along with the edge minimization algorithm by variable grouping. Table I also shows that edge reduction quickly reduces the number of edges.

Table II shows the analysis times when applying the two analysis methods to various EVMDDs. In the analysis using RGEVMDDs, probabilities of shared edges have to be computed before applying the analysis algorithms, and thus, this makes analysis slightly longer when the number of redundant edges is small, like the analysis of a system with $n=15$ and $m=100,000$. But, in many cases, edge reduction makes analysis faster. It can shorten analysis time of optimized GEVMDDs by up to $30 \%$.

\section{COnClusion And COMMEnTs}

This paper proposed a new reduction rule for EVMDDs that reduces redundant edges to speed up the analysis of MSSs. Since analysis time of MSSs depends on the number of edges in an EVMDD, the proposed edge reduction rule can also reduce analysis time. Experimental results show that the proposed rule works more effectively when it is used along with the edge minimization algorithm by variable grouping, and it reduces the number of edges by up to $30 \%$. This results in reducing analysis time of MSSs by up to $30 \%$.

\section{ACKNOWLEDGMENTS}

This research is partly supported by the JSPS Grantin-Aid for Scientific Research (C), (No. 25330071), 2014, and Hiroshima City University Grant for Special Academic Research (General Studies), 2014.

\section{REFERENCES}

[1] S. V. Amari, L. Xing, A. Shrestha, J. Akers, and K. S. Trivedi, "Performability analysis of multistate computing systems using multivalued decision diagrams," IEEE Trans. Comput., Vol. 59, No. 10, pp. 1419-1433, Oct. 2010.

[2] J. D. Andrews and S. J. Dunnett, "Event-tree analysis using binary decision diagrams," IEEE Transactions on Reliability, Vol. 49, No. 2, pp. 230-238, June 2000.

[3] Y.-R. Chang, S. V. Amari, and S.-Y. Kuo, "Reliability evaluation of multi-state systems subject to imperfect coverage using OBDD," the Pacific Rim International Symposium on Dependable Computing (PRDC'02), pp. 193-200, 2002.

[4] Y.-R. Chang, S. V. Amari, and S.-Y. Kuo, "OBDD-based evaluation of reliability and importance measures for multistate systems subject to imperfect fault coverage," IEEE Transactions on Dependable and Secure Computing, Vol. 2 , No. 4, pp. 336-347, 2005.

[5] S. A. Doyle, J. B. Dugan, and F. A. Patterson-Hine, "A combinatorial approach to modeling imperfect coverage," IEEE Transactions on Reliability, Vol. 44, No. 1, pp. 87-94, Mar. 1995.

[6] S. A. Doyle and J. B. Dugan, "Dependability assessment using binary decision diagrams (BDDs)," 25th International Symposium on Fault-Tolerant Computing (FTCS), pp. 249258, June 1995.

[7] T. Kam, T. Villa, R. K. Brayton, and A. L. SangiovanniVincentelli, "Multi-valued decision diagrams: Theory and applications," Multiple-Valued Logic: An International Journal, Vol. 4, No. 1-2, pp. 9-62, 1998.

[8] A. Lisnianski and G. Levitin, Multi-State System Reliability -Assessment, Optimization and Applications, World Scientific 2003.

[9] A. Lisnianski, I. Frenkel, and Y. Ding, Multi-State System Reliability Analysis and Optimization for Engineers and Industrial Managers, Springer 2010.

[10] T. W. Manikas, M. A. Thornton, and D. Y. Feinstein, "Using multiple-valued logic decision diagrams to model system threat probabilities," 41th International Symposium on Multiple-Valued Logic, pp. 263-267, May 2011.

[11] S. Nagayama and T. Sasao, "On the optimization of heterogeneous MDDs," IEEE Trans. on CAD, Vol. 24, No. 11, pp. 1645-1659, Nov. 2005.

[12] S. Nagayama, T. Sasao, and J. T. Butler, "A systematic design method for two-variable numeric function generators using multiple-valued decision diagrams," IEICE Trans. on Information and Systems, Vol. E93-D, No. 8, pp. 2059-2067, Aug. 2010. 
Table I

NUMBER OF EDGES IN EVMDDS FOR $m$-STATE SYSTEMS WITH $n$ 3-STATE COMPONENTS.

\begin{tabular}{|c|c|c|c|c|c|c|c|c|c|c|}
\hline \multirow[t]{2}{*}{$n$} & \multirow[t]{2}{*}{$m$} & \multicolumn{7}{|c|}{ Number of edges } & \multicolumn{2}{|c|}{ Computation time (sec.) } \\
\hline & & EVMDD & REVMDD & Ratio1 & GEVMDD & RGEVMDD & Ratio2 & Ratio3 & Grouping & Reduction \\
\hline 5 & 3 & 27 & 22 & $81 \%$ & 27 & 19 & $70 \%$ & $70 \%$ & ${ }^{*}<0.01$ & ${ }^{*}<0.01$ \\
\hline 5 & 10 & 51 & 42 & $82 \%$ & 48 & 39 & $76 \%$ & $81 \%$ & $*<0.01$ & $*<0.01$ \\
\hline 10 & 3 & 42 & 34 & $81 \%$ & 42 & 32 & $76 \%$ & $76 \%$ & $*<0.01$ & $*<0.01$ \\
\hline 10 & 10 & 168 & 134 & $80 \%$ & 162 & 120 & $71 \%$ & $74 \%$ & $*<0.01$ & $*<0.01$ \\
\hline 10 & 100 & 792 & 662 & $84 \%$ & 750 & 531 & $67 \%$ & $71 \%$ & $*<0.01$ & $*<0.01$ \\
\hline 10 & 1,000 & 2,718 & 2,548 & $94 \%$ & 2,364 & 2,171 & $80 \%$ & $92 \%$ & $*<0.01$ & $*<0.01$ \\
\hline 15 & 3 & 87 & 66 & $76 \%$ & 87 & 64 & $74 \%$ & $74 \%$ & $*<0.01$ & ${ }^{*}<0.01$ \\
\hline 15 & 10 & 312 & 250 & $80 \%$ & 309 & 246 & $79 \%$ & $80 \%$ & $*<0.01$ & $*<0.01$ \\
\hline 15 & 100 & 2,121 & 1,696 & $80 \%$ & 2,076 & 1,571 & $74 \%$ & $76 \%$ & $*<0.01$ & ${ }^{*}<0.01$ \\
\hline 15 & 1,000 & 10,083 & 8,322 & $83 \%$ & 9,597 & 7,164 & $71 \%$ & $75 \%$ & ${ }^{*}<0.01$ & ${ }^{*}<0.01$ \\
\hline 15 & 10,000 & 34,419 & 31,608 & $92 \%$ & 31,212 & 28,234 & $82 \%$ & $90 \%$ & 0.01 & 0.01 \\
\hline 15 & 100,000 & 188,274 & 182,748 & $97 \%$ & 159,768 & 153,216 & $81 \%$ & $96 \%$ & 0.06 & 0.02 \\
\hline
\end{tabular}

Ratio2: RGEVMDD / EVMDD × $100(\%)$

Ratio1: REVMDD / EVMDD × $100(\%)$

* < : It was shorter than $10 \mathrm{msec}$., but could not be obtained precisely due to precision of the timer.

Table II

COMPUTATION TIMES FOR ANALYSIS OF $m$-STATE SYSTEMS WITH $n$ 3-STATE COMPONENTS.

\begin{tabular}{|c|c|c|c|c|c|c|c|c|c|c|c|}
\hline \multirow[t]{2}{*}{$n$} & \multirow[t]{2}{*}{$m$} & \multicolumn{5}{|c|}{ Computation time of bottom-up method [13] ( $\mu$ sec.) } & \multicolumn{5}{|c|}{ Computation time of hybrid method [14] ( $\mu$ sec.) } \\
\hline & & EVMDD & GEVMDD & RGEVMDD & Ratio1 & Ratio2 & EVMDD & GEVMDD & RGEVMDD & Ratio1 & Ratio2 \\
\hline 5 & 3 & 0.96 & 0.98 & 0.77 & $80 \%$ & $\overline{79 \%}$ & 1.77 & $\overline{1.12}$ & 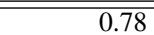 & $\overline{44 \%}$ & $\overline{70 \%}$ \\
\hline 5 & 10 & 2.24 & 2.06 & 1.85 & $83 \%$ & $90 \%$ & 4.76 & 3.13 & 2.42 & $51 \%$ & $77 \%$ \\
\hline 10 & 3 & 1.55 & 1.56 & 1.37 & $88 \%$ & $88 \%$ & 2.74 & 1.68 & 1.24 & $45 \%$ & $74 \%$ \\
\hline 10 & 10 & 6.61 & 6.39 & 5.30 & $80 \%$ & $83 \%$ & 12.14 & 7.53 & 5.59 & $46 \%$ & $74 \%$ \\
\hline 10 & 100 & 42.32 & 33.29 & 26.22 & $62 \%$ & $79 \%$ & 69.60 & 44.30 & 32.65 & $47 \%$ & $74 \%$ \\
\hline 10 & 1,000 & 258.74 & 155.89 & 151.90 & $59 \%$ & $97 \%$ & 360.14 & 230.27 & 199.19 & $55 \%$ & $87 \%$ \\
\hline 15 & 3 & 3.36 & 3.11 & 2.74 & $82 \%$ & $88 \%$ & 5.95 & 4.21 & 3.11 & $52 \%$ & $74 \%$ \\
\hline 15 & 10 & 12.18 & 12.48 & 10.90 & $89 \%$ & $87 \%$ & 21.14 & 14.61 & 11.42 & $54 \%$ & $78 \%$ \\
\hline 15 & 100 & 95.90 & 86.76 & 78.44 & $82 \%$ & $90 \%$ & 148.64 & 102.59 & 79.09 & $53 \%$ & $77 \%$ \\
\hline 15 & 1,000 & 652.68 & 500.55 & 442.38 & $68 \%$ & $88 \%$ & 930.28 & 628.26 & 480.47 & $52 \%$ & $76 \%$ \\
\hline 15 & 10,000 & $3,253.00$ & $2,397.00$ & $2,328.00$ & $72 \%$ & $97 \%$ & $5,408.00$ & $3,753.00$ & $3,169.00$ & $59 \%$ & $84 \%$ \\
\hline 15 & 100,000 & $55,097.00$ & $18,257.00$ & $19,465.00$ & $35 \%$ & $107 \%$ & $24,238.00$ & $15,845.00$ & $14,940.00$ & $62 \%$ & $94 \%$ \\
\hline
\end{tabular}

Ratio1: RGEVMDD / EVMDD × $100(\%)$ Ratio2: RGEVMDD / GEVMDD × $100(\%)$

Very short computation times are average times obtained by running the same computation $1,000,000$ times, and dividing its total time by $1,000,000$.

[13] S. Nagayama, T. Sasao, and J. T. Butler, "EVMDD-based analysis and diagnosis methods of multi-state systems with multi-state components," Journal of Multiple-Valued Logic and Soft Computing, Vol.22, No.1-2, pp.59-78, Feb. 2014.

[14] S. Nagayama, T. Sasao, J. T. Butler, M. A. Thornton, and T. W. Manikas, "Analysis methods of multi-state systems partially having dependent components using multiple-valued decision diagrams," 44th International Symposium on MultipleValued Logic, pp.190-195, May 2014.

[15] S. Nagayama, T. Sasao, J. T. Butler, M. A. Thornton, and T. W. Manikas, "On optimizations of edge-valued MDDs for fast analysis of multi-state systems," IEICE Transactions on Information and Systems, Vol.E97-D, No.9, pp.2234-2242, Sept. 2014.

[16] J. E. Ramirez-Marquez and D. W. Coit, "Composite importance measures for multi-state systems with multi-state components," IEEE Transactions on Reliability, Vol. 54, No. 3, pp. 517-529, Sept. 2005.

[17] A. Shrestha, L. Xing, and Y. Dai, "MBDD versus MMDD for multistate systems analysis," IEEE International Symposium on Dependable, Autonomic and Secure Computing, pp. 172180, 2007.

[18] M. A. Thornton, T. W. Manikas, S. A. Szygenda, and S. Nagayama, "System probability distribution modeling using MDDs," 44th International Symposium on Multiple-Valued
Logic, pp. 196-201, May 2014.

[19] M. Veeraraghavan and K. S. Trivedi, "Combinatorial algorithm for performance and reliability analysis using multistate models," IEEE Trans. on Computers, Vol. 43, No. 2, pp. 229 234, Feb. 1994.

[20] L. Xing and J. B. Dugan, "Dependability analysis using multiple-valued decision diagrams," Proc. of 6th International Conference on Probabilistic Safety Assessment and Management, June 2002.

[21] L. Xing and Y. Dai, "A new decision-diagram-based method for efficient analysis on multistate systems," IEEE Transactions on Dependable and Secure Computing, Vol. 6, No. 3, pp. 161-174, 2009.

[22] E. Zaitseva and V. Levashenko, "Multi-state system analysis based on multiple-valued decision diagram," Journal of Reliability and Statistical Studies, Vol. 5, pp. 107-118, 2012.

[23] X. Zang, D. Wang, H. Sun, and K. S. Trivedi, "A BDD-based algorithm for analysis of multistate systems with multistate components," IEEE Transactions on Computers, Vol. 52, No. 12, pp. 1608-1618, Dec. 2003.

[24] Z. Zhou, G. Jin, D. Dong, and J. Zhou, "Reliability analysis of multistate systems based on Bayesian networks," Proc. of the 13th International Symposium and Workshop on Engineering of Computer Based Systems, pp. 344-352, March 2006. 